AN. MED. INTERNA (Madrid) Vol. 19, N. ${ }^{\circ} 11$, pp. 589-593, 2002

\title{
Síndrome del pasajero de clase económica
}

\author{
F. RÍOS TEJADA, F. VILLEGAS FERNÁNDEZ*, J. A. AZOFRA GARCÍA, \\ L. CALLOL SÁNCHEZ* \\ Servicio Medicina Aeroespacial (CIMA). *Servicio de Neumología. Hospital \\ Universitario del Aire. Madrid
}

\author{
ECONOMIC CLASS SYNDROME
}

\section{RESUMEN}

El grado de confort, seguridad y rapidez conseguido en el transporte por vía aérea, hacen de éste medio un cada vez mas popular sistema de transporte, que es utilizado por un gran número de pasajeros. La horquilla de edad en el pasaje se ha ampliado y la accesibilidad a un espectro social cada vez mas extenso, ha hecho que el número de pasajeros/año se multiplique.

Durante los últimos meses y fundamentalmente producido por la muerte de una pasajera como consecuencia de un tromboembolismo pulmonar (TEP) durante un viaje desde Australia a Europa, se ha discutido sobre el denominado Síndrome del Pasajero de Clase Turista o Económica, en numerosos foros internacionales y se han publicado datos todavía incompletos sobre éste problema.

Muchas preguntas todavía no han respondido de acuerdo a una base científica ¿Cuántos casos?, ¿Se asocia al vuelo?, ¿Cómo podemos prevenirlo?

En este trabajo se analiza la incidencia según la bibliografía consultada, así como aspectos epidemiológicos, fisiopatológicos, factores de riesgo, diagnóstico, probabilidad y medidas preventivas que por parte del usuario, compañías y autoridades aeronáuticas se deberían establecer para evitar en la medida de los posible ésta complicación.

Finalmente se discute las bases fisiopatológicas en las que se desarrolla la trombosis venosa profunda (TVP) y el tromboembolismo pulmonar, tratando de definir éste síndrome como consecuencia de factores relacionados con el medio aeronáutico y en el contexto eventual de su aparición durante el vuelo o como consecuencia directa de éste.

PALABRAS CLAVE: Tromboembolismo pulmonar. Transporte aéreo de pasajeros. Trombosis venosa profunda. Síndrome clase económica.

\section{ABSTRACT}

Comfort, safety and time saving makes air travel more and more popular and widely use by an increased number of people in a range of extended age frame. Recent concerns in the global news have again rai sed an issue about the association of thromboembolic disease and air travel.

Although there are numerous studies in the literature examining venous thromboembolic disease (VTE), variability of population demo graphics makes difficult a right and prospective assessment in the context of the cabin environment and air travel. Literature review clearly demonstrate that VTE is multicausal in nature, resulting in differing etio logic and predisposing factors across different age groups. Many ques tions are still to be answered, How many cases?, Direct association to Air Travel?, How to prevent it in the aviation environment?

In this paper we analize the incidence of VTE and some additional epidemiological aspects, risk and predisposing factors, diagnostic, and preventive measures given by the airline, the user, aviation authorities, aeromedical especialist in order to cope this complication.

Finally we discuss the physiophatological bases in which VTE is developed and related considerations of this symdrome in the context of the aviation environment.

KEY WORDS: Pulmonary embolism. Air travel. Deep venous thrombo sis. Economic class syndrome.

Rios Tejada F, Villegas Fernández F, Azofra García JA, Callol Sánchez L. Síndrome del pasajero de clase económica. An Med Interna (Madrid) 2002; 19: 589-593.

\section{INTRODUCCIÓN}

Viajar por vía aérea se ha convertido en una actividad habitual, frecuente y que básicamente responde a dos razones, trabajo o simplemente placer o turismo. El grado de confort, seguridad y rapidez conseguido para elegir este medio de transporte como el más idóneo hacen que un número cada vez mayor de pasajeros utilizen el transporte aéreo.
Pero esta situación tan sencilla debe considerarse en el amplio contexto de una actividad que necesariamente se relaciona con la vida cotidiana del sujeto y dónde factores como la edad, antecedentes médicos o quirúrgicos, tratamiento médico habitual, actividad realizada previa al vuelo, duración del mismo o grado de familiarización con el vuelo pueden ser cruciales en el momento de valorar un posible evento médico en el transcurso del viaje por vía aérea.

Trabajo aceptado: 4 de marzo de 2002

Correspondencia: F. Ríos Tejada. Jefe Servicio de Medicina Aeroespacial. C.I.M.A. Arturo Soria, 82.28027 Madrid. e-mail: francisco.rios@aero.cima.es 
Otros factores dependientes del diseño del compartimento de pasajeros, distancia entre asientos, pureza del aire ambiente, presurización de la cabina, presencia de humo procedente de asientos de fumadores (en aquellos vuelos dónde aún esté permitido), se han esgrimido como elementos a considerar en la valoración global del grado de confort y seguridad que puede aportar al viajero por via aérea.

Además la accesibilidad del viaje por vía aérea gracias a programas sociales, abaratamiento de precios y aproximación de las compañías aéreas a potenciales usuarios sin límite de edad hacen que la horquilla de edad se haya incrementado y cada vez más veamos pasajeros de la llamada tercera edad volando a destinos antes sin duda insospechados.

Durante los últimos meses y fundamentalmente por el impacto producido por la muerte de Emma Christofferson, después de realizar un vuelo desde Australia a Londres y como consecuencia de un Embolismo Pulmonar, complicación grave de la Trombosis Venosa Profunda, se ha discutido mucho sobre el mal llamado Síndrome del Pasajero de Clase Económica, asociando dicho proceso a una inmovilización prolongada consecuencia de un viaje por vía aérea de larga duración. Algunas preguntas que saltan a nuestra mente podrían ser ¿Cuántos casos se han registrado en vue1o?, ¿La Trombosis Venosa Profunda (TVP) y su complicación mas importante, el Tromboembolismo Pulmonar (TEP) se asocia al vuelo?, y si es así ¿Cómo podemos prevenirlo?.

No obstante estas peculiaridades la incidencia de TVP en la población general se ha establecido en un 1/1000 (1), otros autores como Kesteven (2), señalan cifras del 1,6-1,8/1000. teniendo como patrón el factor edad se podrían establecer cifras de 1/10.000 en personas jóvenes y de 3-5/1000 en edades superiores a los 60 años.

A pesar de la reciente presencia en la literatura médica de numerosos trabajos la variabilidad demográfica utilizada hace poco viable la realización de un metaanálisis $(3,4)$.

La presencia de TVP es de origen multicausal como consecuencia de diferentes etiologías y factores predisponentes que son más relevantes en relación con la edad, por tanto la comorbilidad del proceso ha de entenderse dentro de este complejo (5), de hecho Kesteven en un reciente trabajo concluye que la mayoría de los casos de TVP asociados a viaje por vía aérea ocurren en aquellos sujetos en los que se han identificado factores de riesgo antes del vuelo (6).

\section{ANTECEDENTES}

La primera referencia que se tiene del problema data de 1940, cuando Simpson describe casos de embolismo pulmonar en sujetos que permanecían largo tiempo en refugios anti bombardeos durante la batalla de Inglaterra, donde la movilidad era casi nula (7).

El término "Síndrome de la Clase Económica" fue descrito por vez primera por Symington y Stack en 1977 (8), para describir los problemas de retorno venoso que se producían en los pasajeros que realizaban viajes por via aérea en asientos con una limitación evidente del espacio y del movimiento de los miembros inferiores.

\section{EPIDEMIOLOGÍA}

La presencia de factores de riesgo vascular que en alguna medida incrementen la posibilidad de desencadenamiento de TVP puede llegar hasta un $20 \%$ de la población general. Al reconocerse la edad como un factor adicional, el incremento de la misma coincide con un aumento en la incidencia.

En relación con el factor vuelo se estima una incidencia de 1/1000/año.

Datos reales no existen, por la dificultad en el diagnóstico y sobre todo por la ausencia de relación causa efecto demostrada, siendo ésta la primera razón para que no existan trabajos de suficiente entidad epidemiológica y que cumplan todos los criterios de un trabajo científico. Kraaijenhagen et al en un trabajo reciente llegan a la conclusión que no existe mayor riesgo de TVP en viajeros por via aérea (9). Recientemente Bendz recoge la posible asociación entre un incremento en la actividad del sistema de coagulación y el medio hipóxico, pero dicho trabajo no es concluyente respecto la hipótesis establecida (10).

\section{DEFINICIÓN}

Dicho término se refiere a las complicaciones vasculares que se relacionan con la inmovilización obligada a la que se ve sometido el pasajero de una aeronave, que durante largos períodos de tiempo compromete el retorno venoso de los miembros inferiores, principalmente cuando éstos se mantienen de forma prolongada sujetos a una restricción en su movilidad, y cuyo factor determinante está en el reducido espacio físico que generalmente permite el diseño de los asientos de las aeronaves en la denominada clase económica o turista.

\section{FISIOPATOLOGÍA}

La presencia de enfermedad tromboembólica clásicamente se ha asociado a la denominada triada de Virchow:

-Enlentecimiento en el retorno venoso.

-Hipercoagulabilidad o cambios en la viscosidad sanguínea.

-Alteraciones de la pared vascular.

Todo ello conduce a una mayor predisposición para la formación de trombos en el sistema venoso profundo de los miembros inferiores y por tanto la consecución de la complicación mas frecuente y grave, el tromboembolismo pulmonar.

El Tromboembolismo Pulmonar es el enclavamiento en las arterias pulmonares de uno o varios embolos procedentes de trombosis del territorio venoso. En más del $90 \%$ de los casos éste embolo procede de venas de los miembros inferiores (mmii), siendo el territorio iliofemoral la fuente embolígena mas frecuente. Por tanto, Trombosis Venosa Profunda (TVP) y Tromboembolismo Pulmonar (TEP) son dos entidades interrelacionadas y constituyen lo que se denomina la Enfermedad Tromboembólica Venosa (ETV). Es clásica el aforismo "Si sospechas TEP, mira las piernas". El 10\% de los casos de TVP se asocia a TEP.

Entre los factores predisponentes de TEP mas frecuentes se encuentran:

-Antecedentes de ETV (TVP y/o TEP).

- Cirugía Ortopédica (prótesis de cadera, rodilla).

-Cirugía Abdominal (tumoral, etc.). 
-Neoplasias.

—Estados de hipercoagulabilidad primarios (déficit de ATIII, déficit de proteína $\mathrm{C}$ o de su cofactor la proteína $\mathrm{S}$, factor Vde Leiden etc.).

-Edad.

-Embarazo.

- Enfermedad tumoral subyacente.

- Enfermedad cardiovascular.

—Enfermedad respiratoria crónica.

-Diabetes.

- Traumatismo mmii.

-Parálisis de mmii.

-Factores genéticos.

- Tratamiento contraceptivo, fundamentalmente estrogénico.

-Inmovilización prolongada.

-Deshidratación.

-Obesidad.

-Enfermedad varicosa.

- Tabaquismo.

-Consumo habitual de alcohol/Alcoholismo. némicos.

-Medicación: corticoides, antiagregantes, hipocolesteri-

—Enfermedades de carácter autoinmune.

Los factores genéticos predisponentes pueden llegar a afectar hasta un 5\% de la población general. Entre las coagulopatias mas frecuentes están, el déficit de antitrombina III, déficit de proteina $\mathrm{S}$, déficit de proteína $\mathrm{C}$, mutación del factor $\mathrm{V}$ o Factor V de Leiden (hasta el $40 \%$ de estos pacientes desarrollan TEP), aumento concentración de Factor VIII, Hiperhomocisteinemia y aumento de Protrombina 20210 A (5).

La historia natural de la enfermedad está ligada a un gran número de factores como los mencionados previamente y muy poco se sabe respecto a su asociación con el viaje por via aérea, pero en cualquier caso mas parece tratarse de una asociación que un elemento causal.

No hay estudios prospectivos que aporten datos científicamente validados como para establecer claramente una asociación causa-efecto entre viaje por vía aérea y presencia de TVP o TEP, dichas líneas de trabajo deberían ser capaces de identificar la incidencia real de la enfermedad en el medio aeronáutico y por tanto su potencial riesgo (11).

\section{FACTORES DE RIESGO}

Se desprenden de la patología secundaria a los factores descritos previamente. Hay que mencionar que algunos de éstos factores pueden ser conocidos por el paciente pero no necesariamente, añadiendo en el caso de que se desarrolle la enfermedad un factor sorpresa adicional. En el párrafo anterior se han descrito los factores predisponentes conocidos que conducen a la presencia de TVP y sus complicaciones (TEP).

Podemos clasificar al pasajero como de bajo, moderado o alto riesgo según los siguientes criterios:

\section{BAJO RIESGO}

-Edad> 40 años.

-Obesidad.

- Sexo femenino.

—Reciente cirugía menor (3 días).

\section{MODERADO RIESGO}

-Presencia de varicosidades en mmii.

-Alteraciones cardiovasculares (ICC, IM reciente- 6 semanas-).

- Tratamiento hormonal o utilización de contraceptivos.

-Policitemia y otras alteraciones en los mecanismos de coagulación.

-Anomalías genéticas o adquiridas que afecten a factores de coagulación.

-Embarazo.

-Puerperio.

-Parálisis mmii.

- Trauma mmii reciente (6 semanas)

- Cirugía en mmii o abdomen.

-Inmovilización prolongada.

-Depleción hidrosalina.

\section{ALTO RIESGO}

-Antecedente de tromboembolismo o historia de TVP.

- Anomalías de la coagulación.

-Cirugía mayor reciente (6 semanas): sobre todo cirugía ortopédica y abdominal por cancer.

-Neoplasia.

- Historia familiar de tromboembolismo.

\section{DIAGNÓSTICO}

El TEP es una entidad difícil de detectar, siendo frecuentes el infradiagnóstico y el sobrediagnóstico. Sólo el 20-30\% de los pacientes fallecidos por TEP fueron diagnosticados correctamente en vida (12). Esta dificultad para el diagnóstico se justifica si tenemos en cuenta que no existe ningún síntoma y/o signo clínico, o datos derivados de las exploraciones complementarias de rutina que sean específicos de la enfermedad, solamente alrededor del $30 \%$ de las arteriografías pulmonares practicadas con fines diagnósticos, confirman la existencia de TEP $(13,14)$. El ECG puede mostrar alteraciones hasta en el $56 \%$ de los casos (15). Los síntomas y signos mas frecuentes son la presencia de disnea $(73 \%)$, dolor torácico pleurítico $(77 \%)$ y taquipnea (70\%). La presencia de alguno o más de estos parámetros tiene una sensibilidad del $97 \%$ para el diagnóstico de TEP, sin embargo su especificidad es inferior al 10\% (16). La exploración clínica de las piernas muestra signos de TVP en aproximadamente un tercio de los pacientes con TEP $(15,17)$.

Aunque no existen algoritmos validados para estimar la probabilidad de TEP hay acuerdo general en que dicha estimación se puede realizar teniendo en cuenta los siguientes parámetros (18):

-Presencia de factores de riesgo para enfermedad tromboembólica venosa.

-Clínicos.

—Radiológicos y gasométricos.

Se podría cuantificar la probabilidad clínica de TEP de la siguiente manera:

-Probabiliadad Alta (80-90\%):

- Factor de riesgo presente.

- Clínica compatible.

- Anomalías radiológicasy gasométricas no explicables por otra entidad clínica. 
-Probabilidad Intermedia (20-70\%):

- Ni alta ni baja probabilidad clínica.

-Probabilidad Baja (1-19\%):

- Factor de riesgo no presente.

- Clínica explicable por otra entidad.

- Anomalías radiológicas o gasométricas explicables por otra entidad.

\section{DIAGNÓSTICO}

Entre los medios diagnósticos a nuestro alcance para valorar o confirmar la presencia de TEP, podemos mencionar:

1. Clínicos.

2. Radiología de tórax convencional.

3. Gasometría arterial.

4. Eco-Doppler Sistema Venoso Profundo miembros inferiores.

5. Flebografía.

6. Arteriografía Pulmonar.

7. Gammagrafía Pulmonar de V/Q.

8. TC torácico helicoidal.

9. Angiografía con resonancia magnética.

10. Determinación de dímero D.

11. Electrocardiograma.

\section{INFORMACIÓN AL PASAJERO}

Recientemente el Comité de Ciencia y Tecnología de la Cámara de los Lores Británica (19) y el Comité Médico de Transporte Aéreo de la Sociedad Americana de Medicina Aeroespacial (20) han publicado documentos "had hoc", que tratan de poner en contexto éste problema sentando las bases para establecer unos criterios y pautas de actuación sobre éste problema y que básicamente inciden en los apartados expuestos en éste memorando, pero siempre teniendo muy presente que su sintomatología puede ser inespecífica y a veces asociado a una patología diferente (21).

Es fundamental que el pasajero antes de iniciar el viaje por vía aérea conozca el medio en que va a realizar el viaje desde un punto de vista genérico y en lo que se refiere al ambiente en cabina. En la cabina debería recibir información clara, concisa y asequible sobre este problema. Si ha sido diagnosticado de alguna afección vascular bien arterial o problema de retorno venoso, lo haga saber a su médico de cabecera o médico especialista en Medicina Aeronáutica quien valorará la indicación o no del vuelo o las medidas de precaución a poner en práctica antes y durante el mismo.

\section{MEDIDAS PREVENTIVAS}

-Por parte del Pasajero:

- Ejercicios físicos recomendados.

- Utilización de medias elásticas.

- Medicación bajo prescripción médica especializada.

- Evitar consumo de alcohol.

- Suficiente hidratación.

- Ingesta de sal.
- Evitar la colocación de equipaje detrás del asiento delantero.

- Evitar quedarse dormido con las piernas en flexión forzada.

- Utilizar prendas amplias y cómodas.

- Por parte de la Compañía Aérea:

- Información clara al pasajero.

- Diseño de los asientos y espacio habitable entre asientos.

- Libertad de movimientos en fases no críticas del vuelo.

- Control bebidas alcohólicas.

- Consumo de tabaco en la cabina (no permitido en la gran mayoría de las compañías).

- Oferta especial al pasajero con antecedentes vasculares.

- Por parte de la Autoridad Aeronáutica Civil:

- Reglamentación respecto a espacio disponible entre asientos.

- Tamaño de los asientos.

- Confort general de la cabina.

\section{CONCLUSIONES}

1. No existen estudios que demuestren irrefutablemente la asociación de TVP o TEP y vuelo sin otros factores de riesgo adicional.

2. Es útil la información profesional y exacta que se haga llegar al pasajero sobre este problema. Para ello tanto desde la Administración e Instituciones Médico-Aeronáuticas, como desde las Compañias Aéreas, Tour Operadores, Agencias de Viaje, Autoridades Aeroportuarias o Sociedades Médicas Científicas es positivo que se divulgue al pasajero la información adecuada.

3. Una denominación mas genérica debería establecerse no necesariamente ligada sólo al transporte aéreo.

4. Es necesaria la puesta en marcha de un estudio prospectivo, donde se recoja la información necesaria sobre el impacto real de éste problema en el medio aeronáutico.

\section{RECOMENDACIONES PRÁCTICAS}

1. Medidas generales aplicables a cualquier pasajero independientemente de sus factores de riesgo:

-Ejercicios de flexión y extensión en la articulación del pie.

-Paseos periódicos por el pasillo de la aeronave.

- Abstenerse bebidas alcohólicas.

-Evitar tabaco.

- Hidratación antes y durante el viaje.

- No llevar ropa ajustada.

- No colocar equipaje entre los asientos.

-Evitar flexión prolongada de las extremidades inferiores.

2. Medidas aplicables a pasajeros con bajo riesgo de TVP: - Medidas generales.

-Medias de compresión elástica gradual.

3. Medidas aplicables a pasajeros con riesgo moderado:

-Medidas generales.

- Consulta con su médico especialista.

-Medias de compresión elástica gradual.

—La utilización de Aspirina o Heparina de bajo peso 
molecular exige la indicación precisa por parte del médico especialista quién valorará el coste/beneficio de dicha medida dentro del contexto clínico del paciente.

4. Medidas aplicables en pasajeros de alto riesgo:

- Medidas generales.

\section{Bibliografía}

1. Lomangino N. Venous Thromboembolic Disease: Risk for passengers and aircrew?. Federal Air Surgeons Medical Bulletin 2001; 01-1.

2. Kesteven PJL. Travellers thrombosis. Thorax 2000; 55: 532-6.

3. F. Rios y J. Azofra. El Síndrome del viajero de clase económica. Medicina Aeroespacial y Ambiental 1998; 3: 148-50.

4. Sahiar F. And Moler S. Economy Class Syndrome. Aviat Space Environ Med. 1994, 65: 957-60.

5. Rosendaal FR. Venous thrombosis: a multicausal disease. Lancet 1999; 1999; 353: 1167-73.

6. Kesteven PJL, Robinson BJ. Clinical risk factors for venous thrombosis associated with air travel. Aviat Space Environ Med 2001; 72 : $125-8$.

7. Simpson K. Shelter deaths from pulmonary embolism. Lancet 1940; 2: 744.

8. Symington IS, Stack BHR. Pulmonary thromboembolism after travel. Br J Chest 1977; 71: 138-40.

9. Kraaijenhagen RA et al. Travel and risk of venous trombosis. Lancet 2000; 356: 1492

10. Bendz B et al. Association between acute hypobaric hypoxia and activation of coagulation human beings. Lancet 2000; 356: 1657-8.

11. Geroulakos $\mathrm{G}$. The risk of venous thromboembolism from air travel. The evidence is only circumstancial. BMJ, 2001; 322: 188.

12. Goldhaber SZ, Strategies for diagnosis. En Pulmonary Embolism and Deep Venous Thrombosis. Goldhaber SZ. Saunders Co. Philadelphia 1985. p. 79-97.
- Consulta con su médico especialista.

- Heparinas de bajo peso molecular, siempre bajo la supervisión del médico especialista.

- Valorar posible contraindicación relativa para realizar un viaje por vía aérea de larga duración.

13. Hull RD, Hirsh J, carter CI, et al. Pulmonary angiography, ventilation lung scanning and venography for clinically suspected pulmonary embolism with abnormal perfusion lung scan. Ann Intern Med 1983; 98: 891-9.

14. The PIOPED Investigators. Value of the ventilation/perfusion scan in acute pulmonary embolism: results of Prospective Investigation of Pulmonary Embolism Diagnosis (PIOPED). Jama 1990; 2263: 2753-9.

15. Stein PD, Terrin ML, Hales CA, et al. Clinical, laboratory, roentgenographic and electrocardiographic findings in patients with acute pulmonary embolism and no pre-existing cardiac or pulmonary disease. Chest 1991; 100: 598-603.

16. Flores Segovia J. Diagnóstico de la embolia de pulmón. Rev Pat Respir 2001: 35-38

17. Hoellerich Vl, Wigton RS. Diagnosing pulmonary embolism using clinical findings. Arch Intern Med 1986; 146: 1699-704

18. Hyers T. Venous thromboembolism. Am J Respir Crit Care Med 1999; 159: $1-14$

19. House of Lords. Session 1999-2000, 5th Report. Select Committee on Science and Technology. Air Travel and Health Nov, 2000.

20. Bagshaw M. Traveler's Thrombosis. Deep Vein Thrombosis associated with Travel. Aerospace Medical association-Air Transport Medicine Committee, Jan 2001.

21. Navarro V, Nieto A, Bernacer B, Borras R, Ferrer C. Pneumocystis carinii pneumonia in AIDS and prolonged fever. An Med Interna (Madrid) 1992; 9: 87-90 